\title{
Evolution of crescent-shaped sand dune under the influence of injected sand flux: scaling law and wind tunnel experiment
}

\author{
ZHANG Yang ${ }^{1}$, WANG Yuan ${ }^{1 *}$, ZHOU Xiaosi ${ }^{1}$, YANG Bin ${ }^{2}$ \\ ${ }^{1}$ Department of Fluid Machinery and Engineering, Xi' an Jiaotong University, Xi' an 710049, China; \\ ${ }^{2}$ School of Chemical Engineering, Northwest University, Xi'an 710069, China
}

\begin{abstract}
This paper studies the evolution of crescent-shaped dune under the influence of injected flux. A scaling law and a wind tunnel experiment are carried out for comparison. The experiment incorporates a novel image processing algorithm to recover the evolutionary process. The theoretical and experimental results agree well in the middle stage of dune evolution, but deviate from each other in the initial and final stages, suggesting that the crescent-shaped dune evolution is intrinsically scale-variant and that the crescent shape breaks down under unsaturated condition.
\end{abstract}

Keywords: crescent-shaped sand dune; scale-invariant model; wind tunnel experiment; image processing

Citation: ZHANG Yang, WANG Yuan, ZHOU Xiaosi, YANG Bin. 2017. Evolution of crescent-shaped sand dune under the influence of injected sand flux: scaling law and wind tunnel experiment. Journal of Arid Land, 9(2): 270-277. doi: $10.1007 / \mathrm{s} 40333-017-0005-7$

\section{Introduction}

Crescent-shaped dune is one of the most ubiquitous and fascinating wind-blown sand structures in nature (Bagnold, 1941; Parteli et al., 2007; Kok et al., 2012). It attracts widespread attention because of its unique structure that was generated under unidirectional wind and its accessibility for experimental and theoretical investigations (Durán et al., 2010; Parteli et al., 2014). Field observations suggest that the turnover time of dunes in nature may range from months to years (Pye and Tsoar, 2009; Ewing et al., 2015) and the evolutionary processes associated with the turnover time lengths are not yet in-depth explored. Several small-scale experiments have been conducted to achieve complete evolutionary process, such as those in wind tunnels (Andreotti et al., 2002; Dauchot et al., 2002; Kroy et al., 2002; Faria et al., 2011) and in water channels (Hersen, 2005; Groh et al., 2008; Franklin and Charru, 2011). Small-scale experiment has advantages in quantifying the influence of various controllable factors. In addition, the evolution of small-scale dunes itself is worth studying because large-scale dunes are originated from much smaller ones that are supposed to be eroded away (Andreotti et al., 2002).

Furthermore, numerical simulation provides great benefits as a supplement to experiments. In current numerical research, the mesoscale aspects of sand flux are of greater significance than the characteristics of grains constituting dunes (Sauermann et al., 2001). The minimal model that analyses the continuous dune profile (Herrmann et al., 2001; Kroy et al., 2002) has been extended to 3D cases (Schwämmle and Herrmann, 2004; Parteli et al., 2014), to multiple dune 
interaction (Durán et al., 2010) and to dunes on Mars (Parteli et al., 2007). The minimal model simulation has obtained dune configurations comparable with natural ones. Nevertheless, this model is inserted with a minimal length in the order of ten meters. The cellular automaton (CA) model (Werner, 1995) has been updated to incorporate saltation and avalanche mechanisms (Katsuki et al., 2005) and the lattice-gas scheme (Narteau et al., 2009), and it has been applied to investigate aeolian landscape development (Baas, 2013) and the saturation mechanism (Gao et al., 2014). However, such model is always inserted with empirical mass transfer laws from multi-scale experiments, such as that among 'interacting particles' (Lima et al., 2002) or that among 'sand body element' (Zheng et al., 2009).

In this study, a small-scale experiment is carried out to study the quantitative influence of injected flux on dune evolution, which is seldom conducted in previous investigations. In contrast to the subaqueous experiment that tends to satisfy the major similarity criteria (Groh et al., 2008), the present wind tunnel experiment includes all aeolian mechanisms (such as saltation), and focuses on small-scale sand structures. Accordingly, a simple scaling law, which is scale-invariant and based merely on mass conservation, is introduced to compare with the experimental results.

\section{Scaling law based on mass conservation}

As shown in Figure 1, a crescent-shaped dune is convex on the windward face and steep and concave on the leeward face. Its crest does not necessarily coincide with the brink. The evolution of a crescent-shaped dune includes downwind migration and within-dune deformation, i.e., the stretching of two horns downwind.

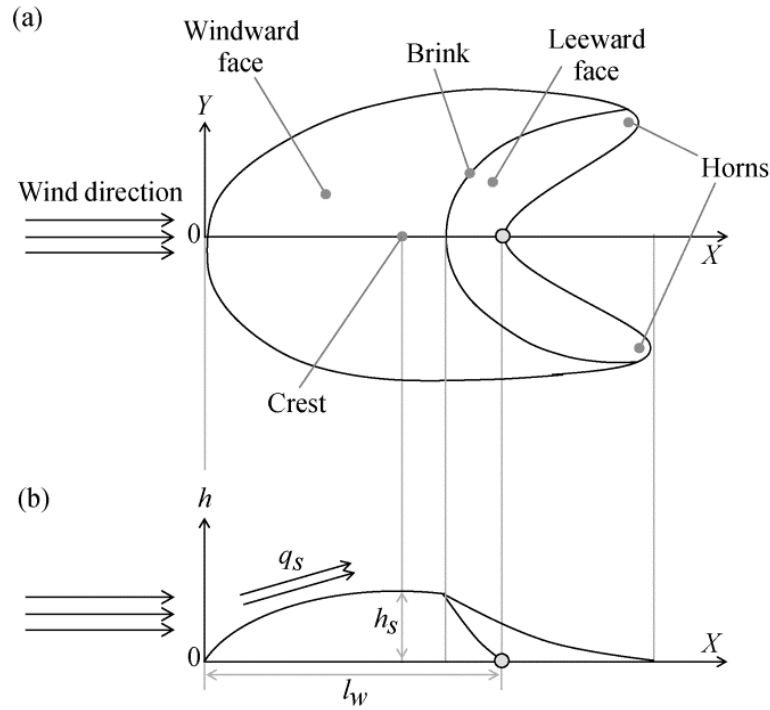

Fig. 1 Crescent-shaped sand dune. (a) Top view of the comprising parts; (b) Side view of two characteristic lengths.

A scale-invariant law is given as follows. As shown in Figure $1 \mathrm{~b}$ and Table 1, consider the longitudinal slice of dune with height profile $h=h(X, t)$, where $X$ and $t$ denote the streamwise direction and time, respectively; and $q_{s}$ is the injected sand flux rate measured in $\mathrm{g} /(\mathrm{m} \cdot \mathrm{s})$. Assume the injected flux to be confined to a layer of thickness $H$, and the averaged flux rate per height unit is $q_{s} / H$. Through a cross section at the abscissa of the maximum height $h_{s}(t)$, sand flux rate can be expressed as $q_{m}=\rho_{s} h_{s} v_{s}$, where $\rho_{s}$ is the sand density; $v_{s}$ is the migrating speed of dune in streamwise direction and is considered constant (Dauchot et al., 2002). Based on mass conservation, $q_{m}$ scales with the sand flux through a section of height $h_{s}$ outside the dune, i.e., $\rho_{s} h_{s} v_{s} \propto q_{s} h_{s} / H$. 
Table 1 Symbol and its corresponding interpretation

\begin{tabular}{|c|c|c|c|c|c|}
\hline Symbol & Interpretation & Symbol & Interpretation & Symbol & Interpretation \\
\hline$A_{1}, A_{2}$ & Positive tuning coefficients & $L_{X 1}$ & $\begin{array}{l}\text { Distance from the } Y \text { axis to } \\
P_{u p}\end{array}$ & $t$ & Time \\
\hline$d_{l e e}$ & $\begin{array}{l}\text { Inter-frame streamwise } \\
\text { displacement of leeward } \\
\text { centre }\end{array}$ & $l_{\text {drag }}$ & $\begin{array}{l}\text { Drag length in wind-blown } \\
\text { sand flux }\end{array}$ & $U_{0}$ & $\begin{array}{l}\text { Mainstream wind speed in } \\
\text { wind tunnel }\end{array}$ \\
\hline$f$ & Image extraction rate & $l_{w}$ & Characteristic dune length & $v_{s}$ & $\begin{array}{l}\text { Streamwise migrating speed } \\
\text { of dune }\end{array}$ \\
\hline$H$ & $\begin{array}{l}\text { Thickness of injected sand } \\
\text { layer }\end{array}$ & $P_{0}$ & $\begin{array}{l}\text { Geometric centre of dune } \\
\text { patch }\end{array}$ & $X$ & Streamwise direction \\
\hline$h$ & Dune height & $P_{i}$ & $\begin{array}{l}\text { Dot on the contour line of } \\
\text { dune patch }(i=1,2, \ldots)\end{array}$ & $Y$ & Transverse direction \\
\hline$h_{0}$ & Initial dune height & $P_{u p}$ & $\begin{array}{l}\text { Most upstream feature point } \\
\text { on dune patch }\end{array}$ & $Z$ & Vertical direction/height \\
\hline$h_{s}$ & Maximum dune height & $q_{s}$ & Injected sand flux rate & $\alpha$ & Angle of repose \\
\hline$L$ & Number of elements in $\left\{P_{i}\right\}$ & $r$ & $\begin{array}{l}\text { Polar radius corresponding } \\
\text { to the dot in }\left\{P_{i}\right\}\end{array}$ & $\Delta$ & $\begin{array}{l}\text { Positive integer between } 0 \\
\text { and } L\end{array}$ \\
\hline$L_{0}$ & $\begin{array}{l}\text { Initial bottom diameter of } \\
\text { sand pile }\end{array}$ & $r^{\prime}$ & Rearranged value of $r$ & $\theta$ & $\begin{array}{l}\text { Polar angle corresponding to } \\
\text { the dot in }\left\{P_{i}\right\}\end{array}$ \\
\hline$L_{X}$ & Image length along the wind & $r_{\max }$ & Maximum value of $r^{\prime}$ & $\rho_{s}$ & Sand density \\
\hline$L_{X 0}$ & $\begin{array}{l}\text { Smallest distance from the } Y \\
\text { axis to dune patch }\end{array}$ & $T$ & Record time of camera & & \\
\hline
\end{tabular}

Now consider the decay rate of mass per spanwise length unit $M \sim \rho_{s} h_{s}{ }^{2}$. According to mass conservation, the balance of erosion and deposition rates must be proportional to the sand flux per height unit times the dune cross section. Moreover, as confined to a layer of thickness $H$, the injected flux is accelerated by a factor of $H /\left(H-h_{s}\right)$ when passing over the dune. In summary, one can obtain:

$$
\frac{d M}{d t} \sim 2 \rho_{s} \frac{d h_{s}}{d \mathrm{t}} h_{s} \propto-\frac{q_{s}}{H} \frac{H}{H-h_{s}} h_{s} .
$$

With two dimensionless quantities $\bar{h}_{s}=h_{s} / h_{s}(0)$ and $\bar{H}=H / h_{s}(0)$, where $h_{s}(0)$ is the initial maximum height, Equation 1 can be rewritten as:

$$
\frac{d \bar{h}_{s}}{d t}\left(\bar{H}-\bar{h}_{s}\right) \propto-\frac{q_{s}}{\rho_{s} h_{s}^{2}(0)} .
$$

Integrating Equation 2 with initial condition $\bar{h}_{s}(0)=1$ and abandoning the 'deposition' situation that $h_{s}$ increases with time, one can obtain:

$$
\frac{h_{s}(t)}{h_{s}(0)}=\bar{H}-\sqrt{A_{1} \frac{t}{t^{*}}+(\bar{H}-1)^{2}} .
$$

Where $t^{*}=\rho_{\mathrm{s}} h_{s}{ }^{2}(0) / q_{s}$ is a normalized time scale suggested by dimensional analysis, and $A_{1}$ is a positive tuning coefficient. One can tell that $d h_{s} / d t<0$ and $d^{2} h_{s} / d t^{2}>0$, which lead to a pattern of concaveness for the function curve $h_{s}=h_{s}(t)$ (Dauchot et al., 2002). This feature is to be recovered experimentally.

\section{Wind tunnel experiment under unsaturated condition}

The above law is scale-invariant, with which small-scale experiments can be used to compare. In this paper, an experiment inside a close-type wind tunnel is carried out to quantitatively evaluate the effect of injected flux.

The crescent-shaped dune produced in this experiment is ephemeral due to the unsaturated status of sand flux. The maximum height of dune $h_{s}$ is difficult to determine from the side view because of the disturbance of the surface saltation layer. Moreover, the characteristic length of dune body $l_{w}$ shown in Figure $1 \mathrm{~b}$ is linearly correlated with the maximum height $h_{s}$ (Hersen et al., 2002; Kroy et al., 2002), thereby giving $l_{w}=A_{2} h_{\mathrm{s}}$, where $A_{2}$ is a positive tuning coefficient. 
Therefore, $l_{w}$ from experiment is selected to compare with the modeled $h_{s}$. The overall length is not selected because it contains the length of horns, which is essentially instable because of the random spread of sand grains avalanching down from the leeward face (Durán et al., 2010). As a result, such instability is particularly significant for small-scale cases. The downstream tip of $l_{w}$ corresponds to the leeward centre (Guignier et al., 2013), which is marked in the circle in Figure 1b. In this experiment, an original image processing algorithm was developed to track the positions of leeward centre from the recorded image sequence.

\subsection{Experimental setup}

A side view of the experimental setup is shown in Figure 2a. The cross section of wind tunnel is $0.5 \mathrm{~m}$ wide and $1.0 \mathrm{~m}$ high. A medium sand sample, with diameter that ranges from 250 to 300 $\mu \mathrm{m}$, is used in the making of the dune. The initial dune is generated by a funnel to have an axisymmetric conical shape (marked in dashed line), and it is placed $8.5 \mathrm{~m}$ downwind of the entrance of wind tunnel. The initial bottom diameter $L_{0}$ and initial height $h_{0}$ of dune ranges from 19.4 to $21.4 \mathrm{~cm}$ and from 7.6 to $8.0 \mathrm{~cm}$, respectively, leading to a stable angle of repose $\alpha \approx 35^{\circ}$. When Equation 3 is combined with $l_{w}=A_{2} h_{s}$, the coefficient $A_{2}$ is no longer needed:

$$
\frac{l_{w}(t)}{L_{0}}=\frac{A_{2} h_{s}(t)}{A_{2} h_{s}(0)}=\bar{H}-\sqrt{A_{1} \frac{t}{t^{*}}+(\bar{H}-1)^{2}} .
$$

Therefore, $l_{w}$ and $h_{s}$ share the scaling law if both are properly normalized. As the wind tunnel is started, a boundary layer with logarithmic wind profile is generated (Fig. 2b), in which the dune moves downwind and deforms towards a crescent shape. A slot is set $1 \mathrm{~m}$ upwind of the edge of conical dune. It is $50 \mathrm{~mm}$ long and $0.5 \mathrm{~m}$ wide, and it is filled with the above mentioned sand sample, thereby having an initial surface level with the wind tunnel floor. The injected flux is generated by constantly bringing sands up to the surface through the slot with a band wrapped around an electrically driven rotating axis. The mainstream wind speed at the height $Z=0.3 \mathrm{~m}$ is set as $U_{0}=7.4 \mathrm{~m} / \mathrm{s}$ in that any sand particle in the slot is immediately blown away once it reaches the surface. Therefore, under this given mainstream wind, $q_{s}$ can be computed together with the rotating speed of the axis and slot area. A glass window is embedded in the wind tunnel ceiling, through which a Phantom digital high-speed camera records the dune evolution from top view with an imaging resolution of 600 pixel $\times 500$ pixel. $T=2 \mathrm{~min}$ is the record time and also about

(a)

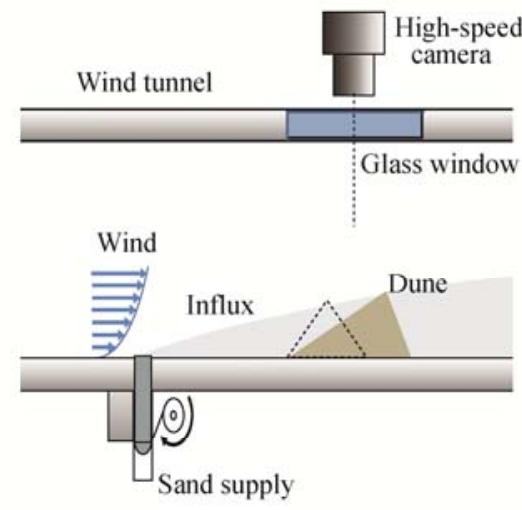

(c)

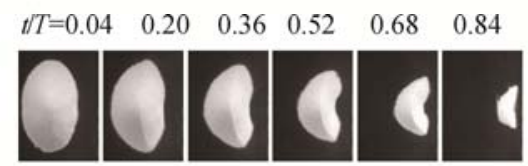

(b)

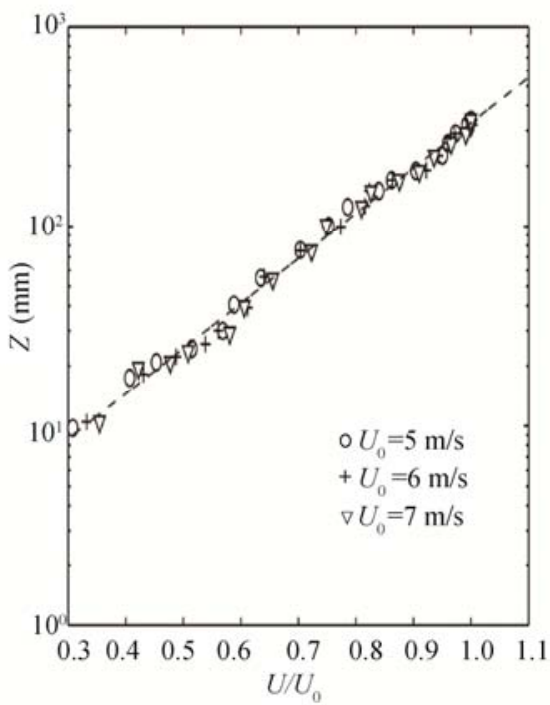

Fig. 2 Small-scale sand dune experiment. (a) Side view of experimental setup; (b) Logarithmic wind profiles measured by hot-wire anemometer $0.2 \mathrm{~m}$ upwind of the slot. $Z$ is the height, $U(Z)$ is the mainstream wind speed, and $U_{0}$ is the mainstream wind speed at $Z=0.3 \mathrm{~m}$; (c) An image sequence recorded from the top view and shown at a relative time interval $t / T=0.16$. 
half of the period for the dune to completely disappear. $f=0.2 \mathrm{~Hz}$ is the image extraction rate. An image sequence extracted from the video (i.e., camera record) is shown in Figure 2c, on which the dune appears as a crescent-shaped patch with a legible brink.

\subsection{Processing of dune image}

Suppose the dune contour line refers to a pixel dot set $\left\{P_{i}\right\}(i=1,2, \ldots)$ and the geometric centre of the entire dune patch is denoted as $P_{0}$. The main idea is to transfer the contour into a 'characteristic curve' according to the polar relationship between $\left\{P_{i}\right\}$ and $P_{0}$, and the extreme points on this curve correspond to the feature points along the contour, which may include the leeward centre. The dune image at $t / T=0.52$ is used as an example. As shown in Figure $3 \mathrm{a}$, a Cartesian coordinate system is set on the contour (in red) with $P_{0}$ as the origin, and each element in $\left\{P_{i}\right\}$ refers to a polar radius $r=r(\theta)$ and a polar angle $\theta \in[-\pi, \pi]$. However, a single $\theta$ value may correspond to two or more elements in $\left\{P_{i}\right\}$, whereas the extreme values can only be found on single-valued function. Therefore, elements in $\left\{P_{i}\right\}$ have to be rearranged first, which can be realized by the following algorithm.

(a)

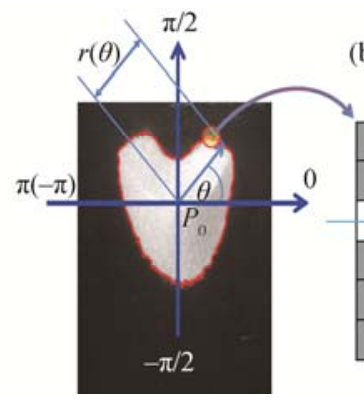

(b)

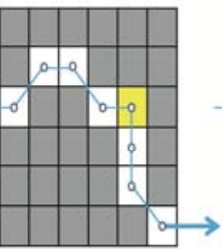

(c)

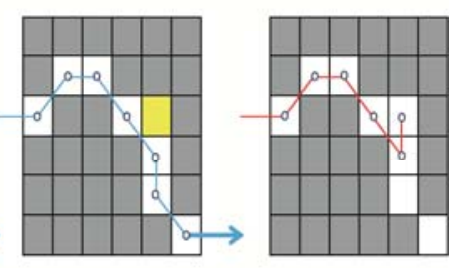

Fig. 3 Processing of dune contour. (a) A Cartesian coordinate system is set on a closed dune contour. Starting from the right horn tip, a dot-chasing route (b) is successfully generated based on the 4-connected priority. A route disregarding this priority may (c) miss a dot or (d) simply terminates.

As shown in Figures $3 \mathrm{~b}-\mathrm{d}$, dots in $\left\{P_{i}\right\}$ are represented by white or yellow squares. Firstly, randomly select a starting dot $P_{1}$ and set a chasing direction (clockwise here). Then $P_{1}$ chases a second dot $P_{2}$, to which it also passes the chasing task to chase $P_{3}$, and so on and so forth. The first rule is that, the chasing route is not allowed to go through a dot more than once. The second rule is that the 4-connected neighbors for a dot $P_{i}$ have priority to be selected as $P_{i+1}$. As the route faces a bifurcation, the dot in yellow is selected because it is a 4-connected neighbor (Fig. $3 b)$. On the contrary, when all 8-connected neighbors have equal opportunity to be selected, the route may either miss the yellow dot (Fig. 3c) or enters a dead end (Fig. 3d) because of the first rule. In sum, this algorithm is capable of rearranging the elements in $\left\{P_{i}\right\}$ without missing any of them.

A rearranged $\left\{P_{i}\right\}$ corresponds to an updated polar radius sequence $\left\{r^{\prime}\right\}$, in which $r^{\prime}$ is no longer a function of $\theta$. Instead, given that the number of elements in $\left\{P_{i}\right\}$ is denoted as $L, r^{\prime}$ becomes a function of the serial number $i$, which takes the integer between 1 and $L$. A normalized relationship between $r^{\prime} / r_{\max }$ and $i / L$ is shown in Figure $4 \mathrm{a}$, given that $r_{\max }$ is the maximum value in $r^{\prime}$. Extreme points must be searched on $r^{\prime} / r_{\max }$, for which the local average is needed and mainly relies on an average period $\Delta \in[0, L]$. Once $\Delta$ is fixed, the local extreme points on the curve (i.e., the feature points along the contour) can be easily found by mathematics. Figure $4 \mathrm{~b}$ shows the variation in searched feature points as a function of the normalized average period $\Delta / L$, where the image length along the wind is denoted as $L_{X}$, the distance from the $Y$ axis to the most upstream feature point $P_{u p}$ is denoted as $L_{X 1}$, and the smallest distance from the $Y$ axis to the entire dune patch is denoted as $L_{X 0}$. When $\Delta / L$ is very small (e.g., $\Delta / L=1 \%$ ), too much details are revealed and excessive feature points are produced. As $\Delta / L$ becomes larger, only the feature points that correspond to the greatest deformation along the contour line can be kept, such as the leeward centre; meanwhile, $P_{u p}$ moves downwind and makes $L_{X 1}$ larger. Figure $4 \mathrm{c}$ shows the 
normalized length $L_{X 1} / L_{X}$ as a function of $\Delta / L$ for six different instants, which indicates that, $P_{u p}$ stays at the leeward centre and makes $L_{X 1} / L_{X}$ stable as $\Delta / L$ is sufficiently large. Hence, given $\Delta / L \geq 50 \%$, the characteristic streamwise length of the dune body can be calculated as $l_{w}=L_{X 1}-L_{X 0}$.

(a)

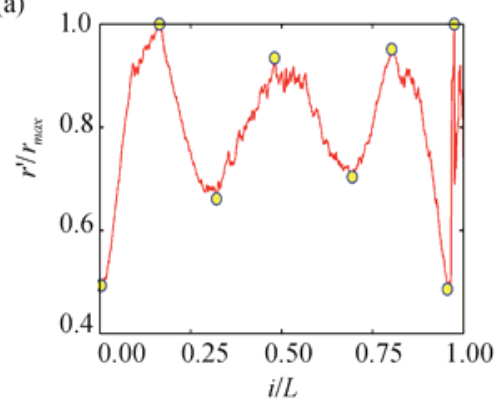

(c)

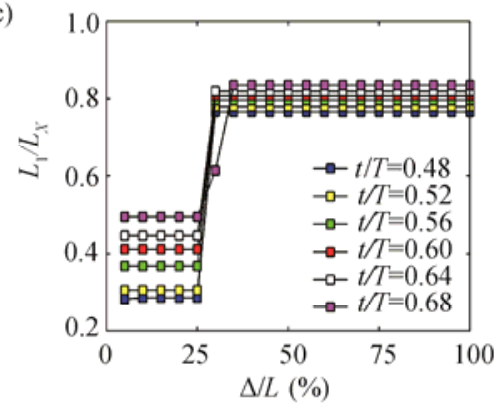

(b)

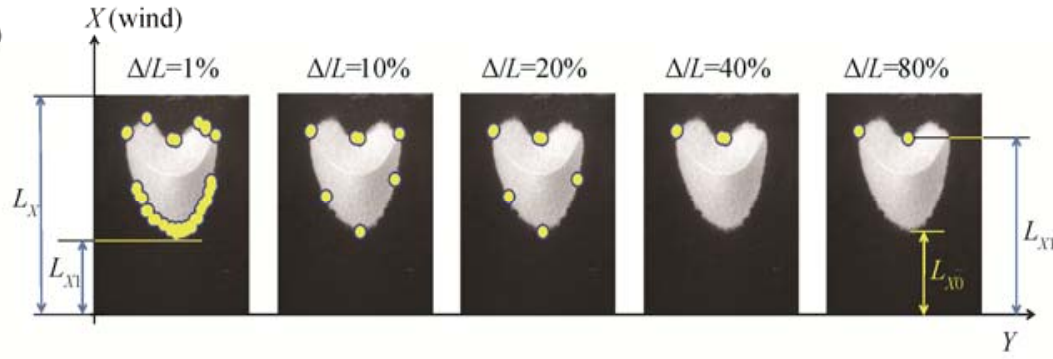

Fig. 4 Calculation of the characteristic streamwise length of dune body for the instant $t / T=0.52$. (a) Updated and normalized polar radius $r^{\prime} / r_{\max }$ as a function of the normalized serial $i / L$ at $\Delta / L=10 \%$; (b) Number of searched feature points decreases along with $\Delta / L$; (c) Normalized distance between the most upstream feature point and the upstream image boundary as a function of $\Delta / L$ for six different instants.

\section{Results and discussion}

Given the intrinsically instable horn length, movement of the located leeward centre is used to evaluate the migrating speed of the dune body. As shown in Figure 5a, $d_{\text {lee }}$ denotes the streamwise displacement of the leeward centre across two consecutive frames, and this parameter is equal to the single-step velocity with forward differential scheme divided by the image extraction rate $f$. Here $f=0.2 \mathrm{~Hz}$ refers to a total of 24 extracted instants (i.e., 23 single-step velocities). Evidently, the inter-frame displacement normalized by $L_{X}$ is stable with time. This finding is in accordance with the above scale-invariant model in which the $2 \mathrm{D}$ dune migrates at a constant speed $v_{s}$.
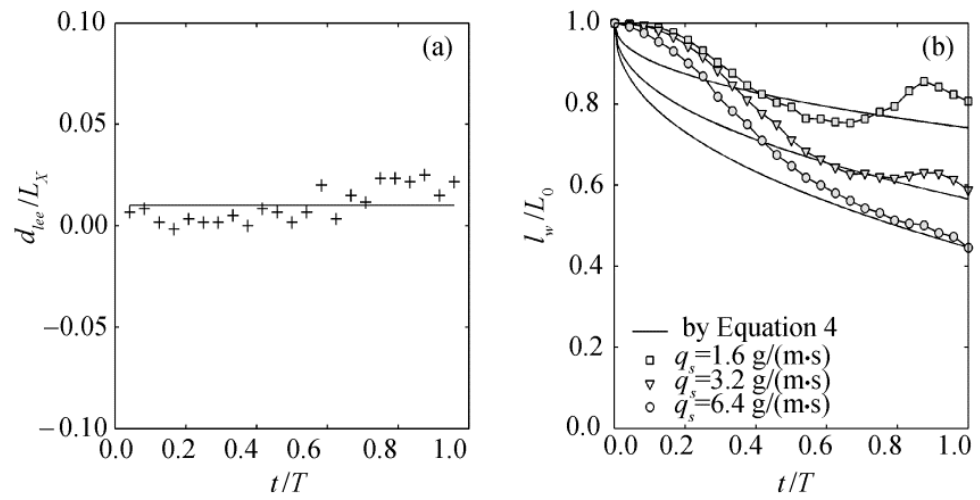

Fig. 5 Parameters describing dune evolution. (a) Inter-frame displacement of the leeward centre along time at $q_{s}=3.2 \mathrm{~g} /(\mathrm{m} \cdot \mathrm{s})$; (b) Comparison between theoretical and experimental results on the length of dune body $l_{w}$, where the injected flux rate increases linearly. 
A total of three runs are conducted, referring to $q_{s}=1.6,3.2$, and $6.4 \mathrm{~g} /(\mathrm{m} \cdot \mathrm{s})$, respectively. In each run, $l_{w}$ is obtained as described in Section 2.2. The corresponding analytical results are obtained using Equation 4. Specifically, a boundary condition is given as $\bar{H} \sim 1$, which agrees with the observation that the injected sand flux extends on a height of the same order of the initial sand dune (Dauchot et al., 2002). Under $q_{s}=1.6 \mathrm{~g} /(\mathrm{m} \cdot \mathrm{s})$, the analytical result is adjusted to fit with the experimental result by the least square principle, leading to a tuning coefficient $A_{1}=1 \times 10^{1}$. This specific value is used for the modeling of the other two runs. The complete result is shown in Figure $5 \mathrm{~b}$ and discussed as follows.

Firstly, the concave feature in Equation 4 is partially recovered by experiment. Specifically, a turning point exists at $t / T=0.4$, before which the modeled $l_{w}=A_{2} h_{s}$ decays much faster than the experimental one. Thus, at the initial stage of dune evolution, $h_{s}$ does not agree very well with $l_{w}$. This phenomenon is possibly caused by the drag length $l_{\text {drag }}$, which is a characteristic length scale of dune formation and relates to the typical saltating distance (Sauermann et al., 2001a). In the current experiment, both $h_{s}$ and $l_{w}$ decrease because sand flux around the dune does not reach the saturated status. $h_{s}$ immediately decays as long as sand grains from the windward face move across the brink, whereas some of them may eventually fall within the leeward domain that exceeds their saltating distances, leading to a slower decrease for $l_{w}$. As the dune continues to evolve (get eroded) and becomes small enough, a sand grain moving across the brink may also leave the dune body, thereby exerting the same strength on the decrease in $l_{w}$ and $h_{s}$. As shown in Figure $5 \mathrm{~b}$, this point is demonstrated by the gradual approach of the experimental result towards the analytical concaveness when $t / T>0.4$. To sum up, the relative size between $l_{\text {drag }}$ and the leeward face length greatly determines the accordance between $l_{w}$ and $h_{s}$. In other words, the evolution of crescent-shaped dunes is essentially scale-variant.

Secondly, as $t / T$ approaches 1 , the experimental result begins to deviate from the modeled one. Under the unsaturated condition, both sand mass and maximum height $h_{s}$ continue to decrease. However, as the dune loses most of its mass, it cannot afford to maintain the crescent form, in which the windward face functions as a shelter for the leeward face. As a result, the dune turns into a ridge (Andreotti et al., 2002), which corresponds to the local increase in $l_{w}$. Moreover, such increase becomes smaller with rising injected flux rate. This finding can be attributed to the fact that a stronger influx drives the overall sand flux around the dune towards the saturated status, thereby diverting the wind energy that is supposed to concentrate on breaking the crescent shape.

The image processing technique in obtaining $l_{w}$ is effective because it takes advantage of the feature of leeward centre such that it represents the greatest deformation along the contour of dune patch. Moreover, the discrepancy in the initial stage of dune evolution $(t / T<0.4)$ can possibly be removed using more effective techniques, i.e., the accurate recognition of dune height from side view, so that a genuine comparison about $h_{s}$ can be realized. Hence, a technique that fully reconstructs the $3 \mathrm{D}$ dune configuration is urgently needed.

\section{Conclusions}

The concaveness of the modelled function curve $h_{s}$ as a function of $t$ has been recovered by experiment in the middle stage of dune evolution based on two conditions. Firstly, the dune should be sufficiently large, which ensures that it does not turn into ridge-like form. Secondly, the dune should be small enough to allow sands moving across the brink to also detach from the dune body, possessing the same strength on the changes in $h_{s}$ and $l_{w}$. Therefore, our model, which intends to bypass the saturation length, demonstrates that at least the drag length is inherited in dune evolution with all sizes. Nevertheless, the above mentioned accordance between theoretical and experimental results in the middle stage of dune evolution suggests that a 2D model still has merit if given a stable crescent shape. 


\section{Acknowledgements}

This work is funded by the National Natural Science Foundation of China (11402190), the China Postdoctoral Science Foundation (2014M552443), and the Natural Science Foundation of Shaanxi Province (2013JQ2001).

\section{References}

Andreotti B, Claudin P, Douady S. 2002. Selection of dune shapes and velocities. Part 1: Dynamics of sand, wind and barchans. The European Physical Journal B-Condensed Matter and Complex Systems, 28(3): 321-339.

Baas A C W. 2013. Modeling aeolian landscapes, In: Shroder J F. Treatise on Geomorphology. San Diego: Academic Press, 313-327.

Bagnold R A. 1941. The Physics of Blown Sand and Desert Dune. New York: Methuen, 39-98.

Dauchot O, Lechénault F, Gasquet C, et al. 2002. "Barchan” dunes in the lab. Comptes Rendus Mécanique, 330(3): $185-191$.

Duran O, Parteli E J R, Herrmann H J. 2010. A continuous model for sand dunes: review, new developments and application to barchans dunes and barchan dune fields. Earth Surface Processess \& Landforms, 35(13): 1591-1600.

Ewing R C, Hayes A G, Lucas A. 2015. Sand dune patterns on Titan controlled by long-term climate cycles. Nature Geoscience, 8(1): 15-19.

Faria R, Ferreira A D, Sismeiro J L, et al. 2011. Wind tunnel and computational study of the stoss slope effect on the aeolian erosion of transverse sand dunes. Aeolian Research, 3(3): 303-314.

Franklin E M, Charru F. 2011. Subaqueous barchan dunes in turbulent shear flow. Part1. Dune motion. Journal of Fluid Mechanics, 675(5): 199-222.

Gao X, Zhang D, Rozier O, et al. 2014. Transport capacity and saturation mechanism in a real-space cellular automaton dune model. Advances in Geosciences, 37: 47-55.

Groh C, Wierschem A, Aksel N, et al. 2008. Barchan dunes in two dimensions: experimental tests for minimal models. Physical Review E, 78(2): 021304.

Guignier L, Niiya H, Nishimori H, et al. 2013. Sand dunes as migrating strings. Physical Review E, 87(5): 052206.

Herrmann H J, Kroy K, Sauermann G. 2001. Saturation transients in saltation and their implications on dune shapes. Physica A: Statistical Mechanics and Its Applications, 302(1-4): 244-254.

Hersen P, Douady S, Andreotti B. 2002. Relevant length scale of barchan dunes. Physical Review Letters, 89(26): 264301.

Hersen P. 2005. Flow effects on the morphology and dynamics of aeolian and subaqueous barchan dunes. Journal of Geophysical Research: Earth Surface, 11(F4): F04S07.

Katsuki A, Nishimori H, Endo N, et al. 2005. Collision dynamics of two barchan dunes simulated using a simple model. Journal of the Physical Society of Japan, 74(2): 538-541.

Kok J F, Parteli E J R, Michaels T I, et al. 2012. The physics of wind-blown sand and dust. Reports on Progress in Physics, 75(10): 106901.

Kroy K, Sauermann G, Herrmann H J. 2002. Minimal model for aeolian sand dunes. Physical Review E, 66(3): 031302.

Lima A R, Sauermann G, Herrmann H J, et al. 2002. Modeling a dune field. Physica A: Statistical Mechanics and its Applications, 310(3-4): 487-500.

Narteau C, Zhang D, Rozler O, et al. 2009. Setting the length and time scales of a cellular automaton dune model from the analysis of superimposed bed forms. Journal of Geophysical Research: Earth Surface, 114(F3): F03006.

Parteli E J R, Duran O, Herrmann H J. 2007. Minimal size of a barchan dune. Physical Review E, 75(1): 011301.

Parteli E J R, Kroy K, Tsoar H, et al. 2014. Morphodynamic modeling of aeolian dunes: Review and future plans. The European Physical Journal Special Topics, 223(11): 2269-2283.

Pye K, Tsoar H. 2009. Aeolian Sand and Sand Dunes. Berlin Heidelberg: Springer.

Sauermann G, Kroy K, Herrmann H J. 2001. Continuum saltation model for sand dunes. Physical Review E, 64(3): 031305.

Schwämmle V, Herrmann H J. 2004. Modeling transverse dunes. Earth Surface Processes \& Landforms, 29(6): 769-784.

Werner B T. 1995. Eolian dunes: computer simulations and attractor interpretation. Geology, 23(12): 1107-1110.

Zheng X J, Bo T L, Zhu W. 2009. A scale-coupled method for simulation of the formation and evolution of aeolian dune field. International Journal of Nonlinear Sciences and Numerical Simulation, 10(3): 387-396. 